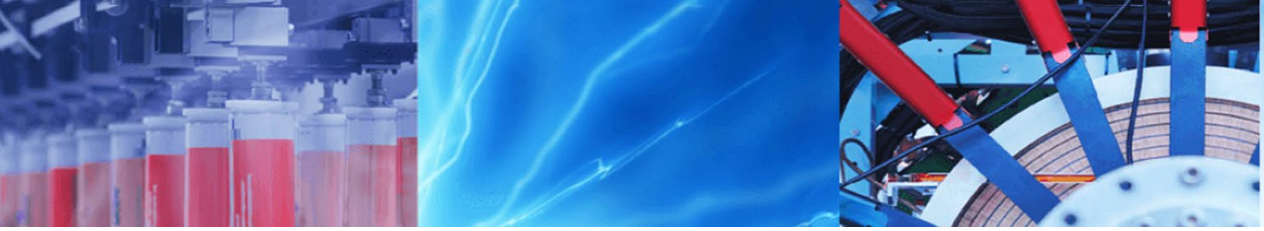

Research Article

\title{
Finite element analysis of elastic-plastic and fracture behavior in functionally graded materials (FGMs)
}

\author{
M. M. Shahzamanian ${ }^{1,2} \cdot$ Amir Partovi $^{1} \cdot$ P. D. $\mathrm{Wu}^{1}$
}

Received: 7 September 2020 / Accepted: 18 November 2020 / Published online: 2 December 2020

(c) Springer Nature Switzerland AG 2020

\begin{abstract}
The effect of material property variation on ductility and fracture strain in functionally graded materials (FGMs) is investigated using the finite element method (FEM) and the Gurson-Tvergaard-Needleman (GTN) model, which is straincontrolled for void nucleating. The material properties of FGMs in the tensile tests are assumed to be represented by a power-law distribution in the thickness direction. A gradation index $(n)$ assigns the material property distribution. The lower and upper surfaces are pure hard $(n=\infty)$ and pure soft $(n=0)$ materials, respectively, in the simulations. The ductility and fracture strain changes with varying gradation index. The development of stress triaxiality slows down in FGMs, and the total void volume fraction is reduced. The analysis is performed for five values of $n$, and the results are discussed numerically. The aim of this study is to help the researchers for better design and fabrication of FGMs. The necking strain in FGMs is calculated and it is found that it increases when the rate of hardening in the effective stress-strain curve increases. Lastly, it can be concluded that gradation index has significant effect on the elastic-plastic and fracture behavior of the functional graded (FG) specimen.
\end{abstract}

Keywords Elastic-plastic response · Fracture · Finite element method (FEM) · Functionally graded materials (FGMs)

\section{Introduction}

There are several methods to increase the ductility of metals such as superimposing hydrostatic pressures as performed for sheets under bending [1] and for rings under compression [2]. However, another method to increase the ductility is cladding the sheet metals. Monolithic materials may not provide the desired properties; Laminated Metal Composites (LMCs) consist of alternating material layers, which exhibit the combined properties of their components to obtain composite properties [3]. In Ref. [4], LMCs can significantly improve many properties, such as fracture toughness [5], fatigue behavior [6], impact behavior [7], and formability [8]. Cladding materials possess the combined strength and ductility of metals [9]. However, sudden material property change in LMCs creates sharp stress distributions at the interface between the material layers. FGMs contain two or more constituents that have volume fraction that varies smoothly and continuously as a function of position along a certain dimension(s) of the structure [10]. The sudden and sharp stress distribution in a particular direction can be eliminated when the material properties vary smoothly and gradually. Figure 1 shows a comparison of thermal stress distribution between a conventional thermal barrier and a thermal barrier made of FGM. The sharp stress distribution is eliminated in FGM. However, to the best of our knowledge, the effect of material property gradation on ductility and fracture strain in FGMs has not been reported in detail elsewhere.

$\triangle$ M. M. Shahzamanian, mmshahzamanian@gmail.com; mshahzam@ualberta.ca| ${ }^{1}$ Department of Mechanical Engineering, McMaster University, Hamilton, ON L8S 4L7, Canada. ${ }^{2}$ Department of Civil and Environmental Engineering, University of Alberta, Edmonton, AB T6G 2R3, Canada. 
Fig. 1 Comparison between a conventional and FGM thermal barrier [16]

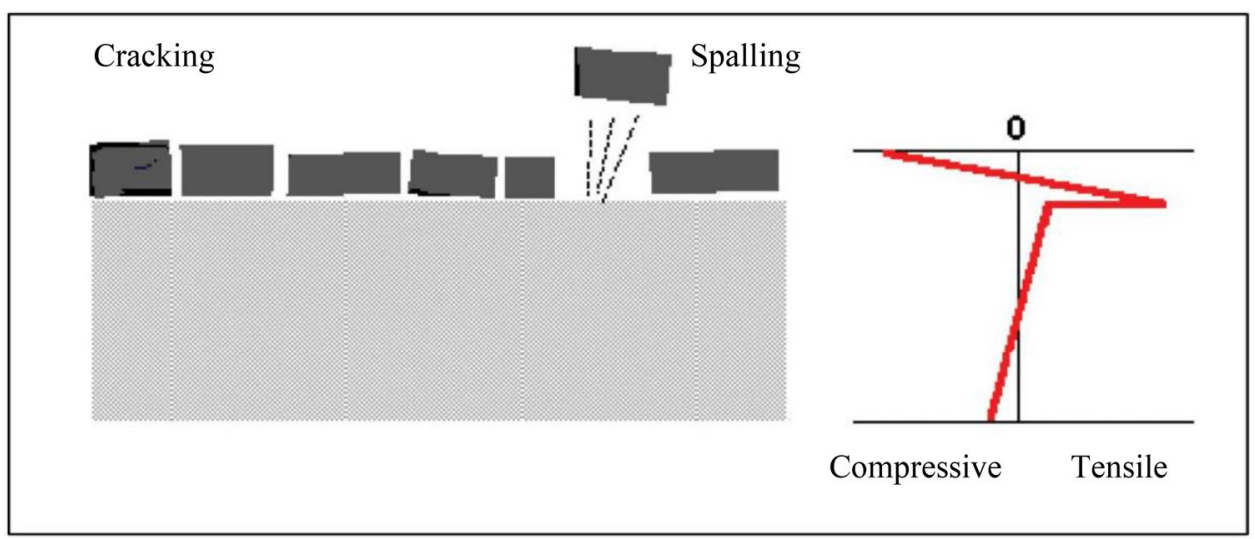

(a) Conventional thermal barrier coating and related failure modes

(b) Thermal stress distribution

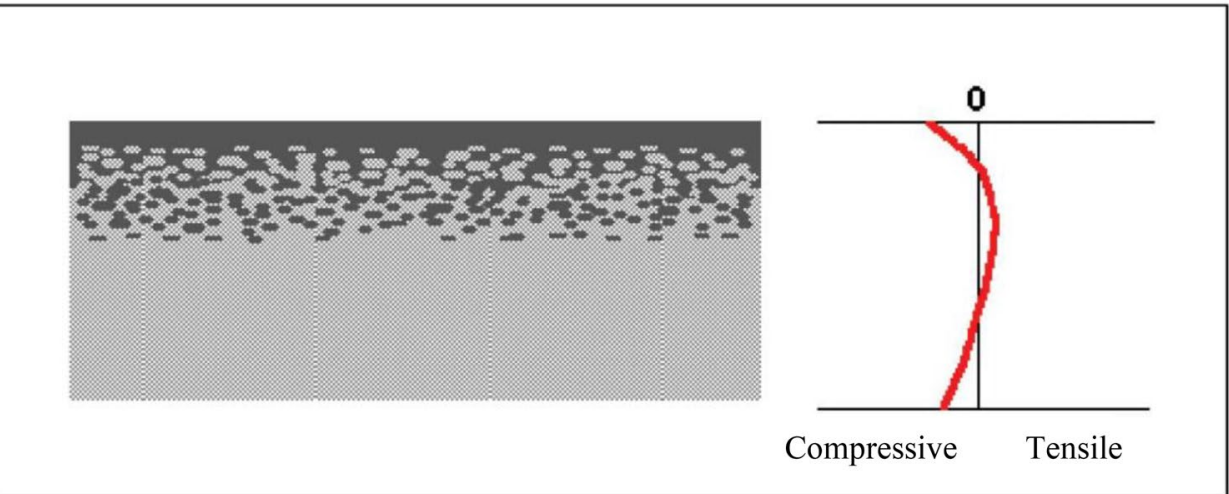

(c) FGM Thermal Barrier Coating

(d) Thermal stress distribution
Many researchers have investigated the behavior of FGMs for instance the static and vibration analysis of FG carbon nanotube reinforced composite in [11] and elastic-plastic response of FGMs under torsion in [12] are performed. Kordkheili and Naghdabadi [13] applied semi-analytical methods to obtain the thermoelastic solutions for axisymmetric FGM rotating disks under plane stress condition. The results are compared with those presented by Durodola and Attia [14] under centrifugal loading. FGMs have also been simulated in finite element (FE) software, such as ANSYS and ABAQUS, and various analyses have been performed. In Ref. [14], the deformation of FG rotating disc was determined using the FEM. In these analyses, the material is subdivided into several layers in a certain direction, and each layer has a different material property following a gradation relation. In previous studies, FG brake discs were simulated in ANSYS when material properties vary in radius [15] and thickness [16] directions, and thermoelastic contact problems were studied. In Ref. [17], the FGM was divided into several layers to investigate the sliding frictional contact problem under the plane strain state with varying shear modulus in the thickness direction. Furthermore, the elastic-plastic response in FGMs is analyzed numerically for various applications. Gunes et al. [18] investigated the elastic-plastic response of FG circular plates under low impact-velocities using FEM. In their model, the stress-strain curve of layers changes from fully plastic material for a ductile metal to linear elastic material for a brittle ceramic following the intermediate law of mixture. Both experimental and numerical studies about the effect of elastic-plastic properties variation on low-velocity impact behavior are performed by Gunes et al. [19]. The FGMs are fabricated using the powder stacking-hot pressing technique experimentally and the Mori-Tanaka scheme is used to determine the elastic-plastic stress-strain curves of the FGMs layer numerically.

For many high-strength sheet materials, including aluminum alloys, which contain a significant amount of second phase particles, micro-voids often result in the vicinity of particles during large plastic deformation during formation. These particle-induced micro-voids are known to localize plastic flow and limit the formability of sheet materials [20]. One of the well-known models of ductile void growth that is often utilized in analyzing large plastic deformation of ductile metallic materials is the Gurson model [21], and its later modification called the GTN 
model, proposed by Tvergaard and Needleman [22]. These models treat voids as spherical cavities and capture their effects on material yield following a modification of the von Mises yield criterion [21].

Many researchers investigated the elastic-plastic response and fracture behavior of cladding metals employing FEM. Chen et al. [23] investigated the effect of cladding a ductile layer in ring and sheet metals under plane strain tension on necking and fracture strains by using the GTN model. In Ref. [23], cladding a ductile ring increased the necking and fracture strains and enhanced the work hardening rate. The necking strain increased following the rule of mixtures, and cladding delayed void nucleation and growth, resulting in significant ductility. The topological arrangement of cladding in the ring was also investigated. Although the topological arrangement of the cladding ring affects the fracture strain, the necking strain is unaffected. The effect of cladding material on bendability is studied in Ref. [24]. Results show that bending fracture strain increases with increased claddingthickness ratio. A comprehensive study is performed to understand the effect of cladding material stress-strain curve on bendability. A previous study [24] has revealed that fracture strain is unchanged when the rate of hardening in the cladding material stress-strain curve is constant for those specific stress-strain curves in cladding and core materials.

In this study, the elastic-plastic response and fracture behavior of FGMs are investigated under plane strain by using the GTN model in ABAQUS/Explicit software. The FGM is simulated using several layers, and each layer has different material properties. The material properties vary in the thickness direction following the gradation relationship, where the surfaces of the specimen are cladding and base materials with parameters used in Ref. [23]. The stress-strain curves and a typical ductile fracture parameter in the GTN model vary from base metal located on the bottom of the specimen to the cladding material located on the top of the specimen. The effect of material property change in thickness direction is analyzed. The results show that ductility increases when using FGMs without having sharp and sudden stress distributions along the direction where material properties vary.

\section{Constitutive model}

The GTN model [22] is used in this study to understand the effect of grading index in FGMs on ductility and fracture strain. Void growth and nucleation in the GTN model create damage growth and it leads to coalescence and eventually ductile fracture in metals. The void growth and void nucleation growth are expressed in Equations (1) and (2), respectively:

$(\dot{f})_{\text {growth }}=(1-f) \mathbf{I}: \mathbf{D}^{P}$

where, $\mathbf{D}^{P}$ is the plastic strain rate.

$(\dot{f})_{\text {strain controlled nucleation }}=\bar{A}_{\overline{\bar{\varepsilon}}}^{P}$

where $\dot{\bar{\varepsilon}}^{P}$ is the effective plastic strain rate. Chu and Needleman [25] suggested that the parameter $\bar{A}$ follows a normal distribution as:

$\bar{A}=\frac{f_{N}}{S_{N} \sqrt{2 \pi}} \exp \left[-\frac{1}{2}\left(\frac{\bar{\varepsilon}^{p}-\varepsilon_{N}}{S_{N}}\right)^{2}\right]$

here, $\varepsilon_{N}$ is the average void nucleating strain, $f_{N}$ is the volume fraction of void nucleating particles, $S_{N}$ is the standard deviation of void nucleating strain.

The void nucleation can also be assumed to be stress-controlled:

$(\dot{f})_{\text {stress controlled nucleation }}=A \dot{\bar{\sigma}}+B \dot{\sigma}_{H}$

where, $A$ and $B$ are taken to depend on the current stress state value of the matrix equivalent plastic strain. In ABAQUS, only the strain-controlled void nucleation is considered and the stress-controlled void nucleation is not available.

The evolution of void volume fraction in the GTN model is the summation of the void growth and void nucleation growth as follows:

$\dot{f}=(\dot{f})_{\text {growth }}+(\dot{f})_{\text {nucleation }}$

In order to consider the effect of coalescence, the following expression [Eq. (6)] for the void volume fraction $\left(f^{*}(f)\right)$ is defined in the GTN model. $f^{*}$ is assumed to be distributed randomly. When $f$ reaches $f_{c^{\prime}}$ coalescence happens and on the contrary fracture happens when $f$ reaches $f_{f}$.

$f^{*}= \begin{cases}f & \text { for } f \leq f_{c} \\ f_{c}+\frac{f_{u}^{*}-f_{c}}{f_{f}-f_{c}}\left(f-f_{c}\right) & \text { for } f>f_{c}\end{cases}$

where, the parameter $f_{u}^{*}=1 / q_{1}$ is defined.

It is to be noted that void growth and nucleation only happen when the stress state of an element is in tension. However, the approximate yield function in the GTN model is as follows:

$\Phi(\sigma, \bar{\sigma}, f)=\frac{\sigma_{e}^{2}}{\bar{\sigma}^{2}}+2 f^{*} q_{1} \cosh \left(\frac{3 q_{2} \sigma_{H}}{2 \bar{\sigma}}\right)-\left[1.0+\left(q_{2} f^{*}\right)^{2}\right]=0$ 
where, $\boldsymbol{\sigma}$ is the macroscopic Cauchy stress tensor and $\sigma_{e^{\prime}} \sigma_{H}$ and $\bar{\sigma}$ are equivalent stress, hydrostatic stress and matrix stress, respectively. Also, $q_{1}$ and $q_{2}$ are the fitting parameters.

The power-law function is used to express the uniaxial elastic-plastic undamaged stress-strain curve for the matrix material as presented in Eq. (8).

$\bar{\sigma}=\left\{\begin{array}{l}E \bar{\varepsilon}, \text { for } \bar{\sigma} \leq \sigma_{y} \\ K \bar{\varepsilon}^{\tilde{n}}, \text { for } \bar{\sigma}>\sigma_{y}\end{array}\right.$

\section{Problem formulation and method of solution}

The schematic of an FG sheet containing 25 layers with length $I_{o}$ and thickness $h$ is shown in Fig. 2 . The sheet is assumed to be sufficiently wide and has no deformation in width direction to consider the plane strain state. A relatively stubby specimen is considered because only the neck region is of interest and displacements are applied on the edges to deform the specimen in Y-direction. The length of the tensile specimen is threefold higher than the initial thickness of the tensile sample, as simulated in Ref. [26]. The layer located on the bottom surface of the specimen is the base material, and the layer located on the top surface is the cladding material. The properties of each layer $(P)$ include elastic-plastic stress-strain curve and average void nucleation strain $\left(\varepsilon_{N}\right)$ change following the power-law distribution in the thickness direction, as follows:

$P(y)=P_{\text {cladding }}((2 y+h) / 2 h)^{n}+P_{\text {base }}\left(1.0-((2 y+h) / 2 h)^{n}\right) ; n \geq 0$

where $P_{\text {cladding }}$ denotes the material property of the top surface (cladding material), and $P_{\text {base }}$ denotes the material properties of the bottom surface (base material) of the tensile test specimen. The grading index $(n)$ indicates the material property variation through the thickness direction $Y$. The other parameters in the GTN model remain the same for every layer.

\section{Results and discussion}

Displacements in the $X$ direction are applied on the opposite surfaces of the FG specimens perpendicular to the $X$ direction while the other directions are constrained. The results are presented in this section and the effect of grading index as well as cladding stress-strain curve on the ductility and fracture strain are discussed.

\subsection{Effect of grading index on ductility and fracture strain}

The elastic-plastic properties of the base and cladding materials are specified as in Refs. [23] and shown in Table 1. The values of the mechanical properties of the matrix material for the GTN model, which is strain-controlled for
Fig. 2 Schematic of an FG tensile test specimen

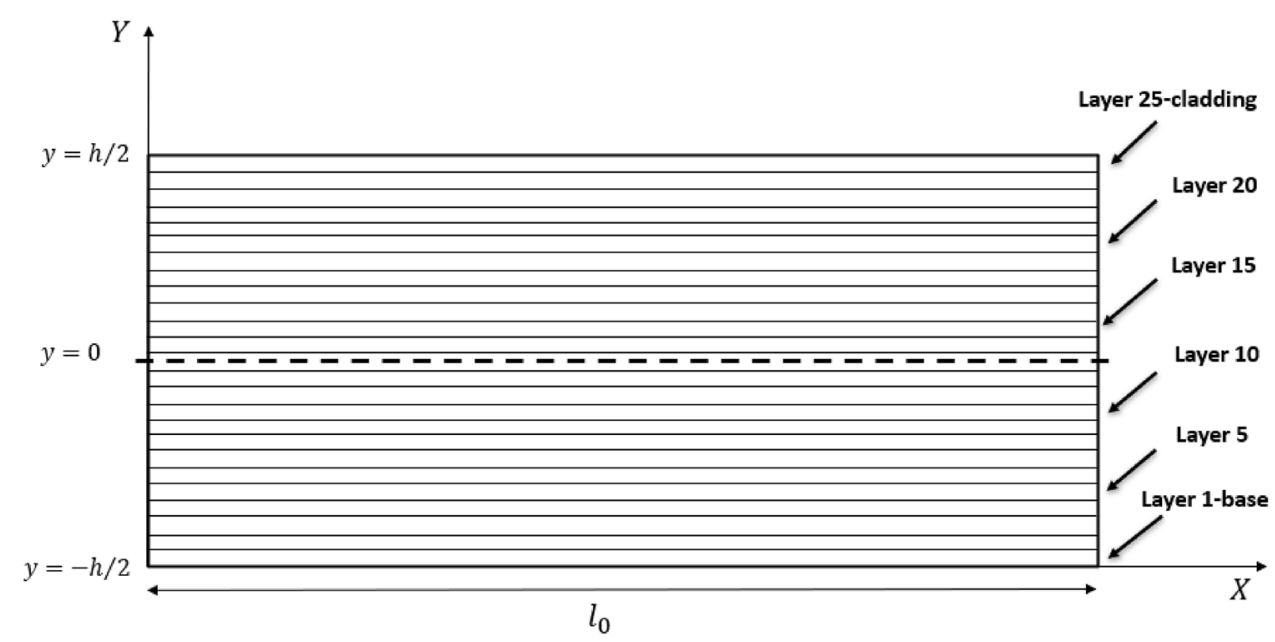

Table 1 Matrix and cladding material constants

\begin{tabular}{llllllllllll}
\hline & $\sigma_{y} / E$ & $v$ & $K$ & $\tilde{n}$ & $q_{1}$ & $q_{2}$ & $f_{N}$ & $\varepsilon_{N}$ & $S_{N}$ & $f_{c}$ & $f_{f}$ \\
\hline Base material & 0.0033 & 0.3 & 414.9 & 0.1 & 1.5 & 1.0 & 0.04 & 0.3 & 0.1 & 0.15 & 0.25 \\
Cladding material & 0.002 & 0.3 & 492.1 & 0.2 & 1.5 & 1.0 & 0.04 & 0.5 & 0.1 & 0.15 & 0.25 \\
\hline
\end{tabular}


void nucleating are taken from Tvergaard and Needleman [22].

In previous studies [23], the purpose of cladding is to enhance formability and ductility. Therefore, the cladding material is assumed to have higher hardening and ductility than the base material. Compared with the base material, the cladding material has relatively lower yield stress but higher hardening and resistance to void nucleation [23]. The values of the material parameters for the cladding material are assumed to be the same as those for the base material, except for $\sigma_{y} / E, K, \tilde{n}$ and $\varepsilon_{N}$. As mentioned previously, the index $n$ in FGMs indicates variations in material properties in the thickness direction. The specimens are fully base material when $n=\infty$ and fully cladding material when $n=0$. In this study, various $n$ values $(0,0.5,1.0$, $5.0, \infty)$ are considered. Figure 3 shows the variation in the true stress-strain curves of FGMs with $n=0.5,1.0,5.0$. The

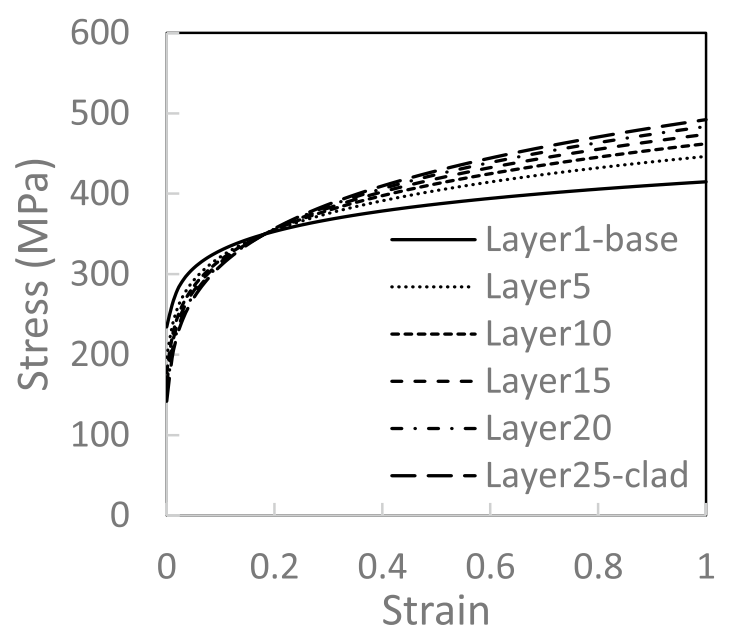

(a) $n=0.5$

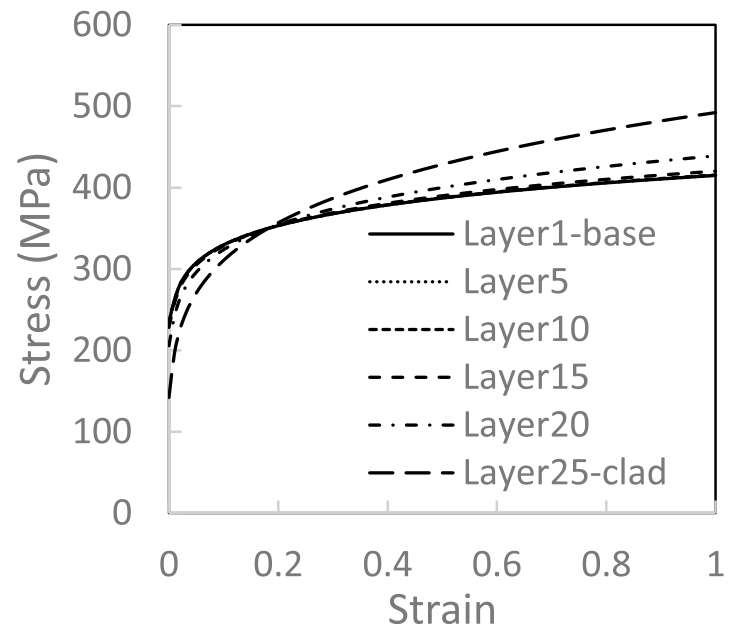

(c) $n=5.0$ stress-strain curves for $n=1.0$ are distributed evenly. The stress-strain curves of the layers are prone to the base material stress-strain curve when $n>1.0$. The stress-strain curves of the layers are prone to the cladding material when $n<1.0$. The effective stresses of each case are calculated following the rule of mixture. Figure $3 \mathrm{~d}$ shows that the effective stress for $n=1.0$ is the average of the two stress-strain curves of the base and cladding materials. The stress-strain curve for case $n=0.5$ is close to that of the cladding material. The stress-strain curve for $n=5.0$ is close to the stress-strain curve of the base material. Figure 4 also shows the variation in $\varepsilon_{N}$ in terms of thickness for various $n$ values. The $\varepsilon_{N}$ value of the layers for $n=1.0$ is distributed evenly. The $\varepsilon_{N}$ values for the FGMs with $n=0.5$ and 5.0 are higher and lower than those for FGM with $n=1.0$, respectively. The average $\varepsilon_{N}$ values for FGMs with $n=0.5$, 1.0 and 5.0 are $0.366,0.4$ and 0.432 , respectively.

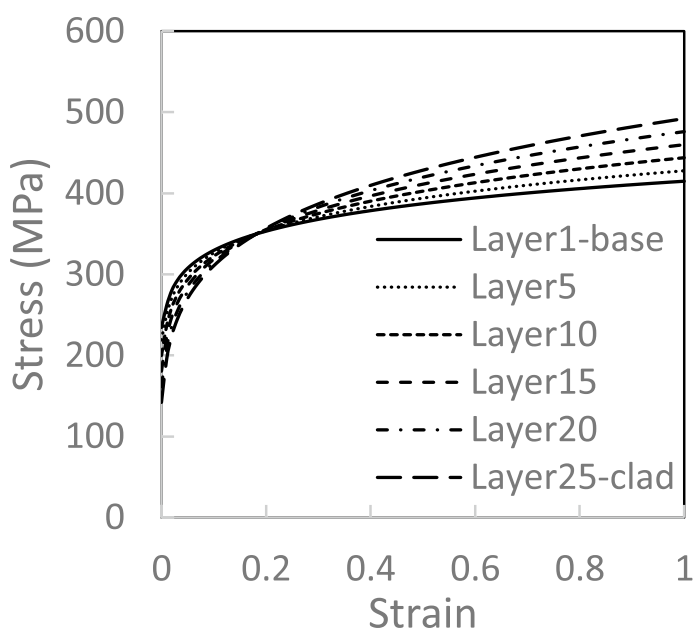

(b) $n=1.0$

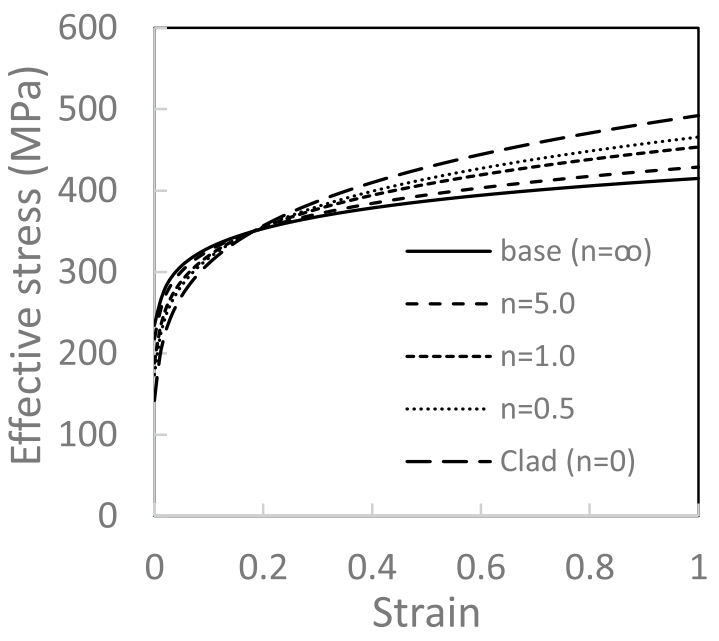

(d) Effective stress-strain curve

Fig. 3 Stress-strain curves in FGM layers for various values; $\mathbf{a} n=0.5 ; \mathbf{b} n=1.0 ; \mathbf{c} n=5.0$ and $\mathbf{d}$ effective stress-strain curves in FGMs 


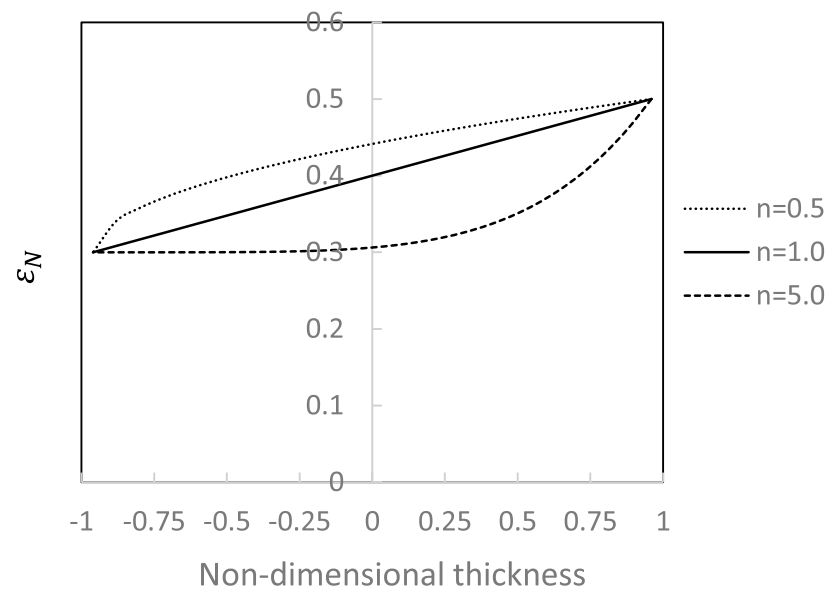

Fig. 4 Average nucleating strain $\left(\varepsilon_{N}\right)$ variation in thickness for various values $n$

This study mainly aims to investigate the effect of material property gradation on the ductility and fracture strain of sheet metals. Thus, the results and conclusions are not exclusively dependent on the values of the material parameters used in this study. This study only explains how the grading index in FGMs affect the ductility and fracture strain.

ABAQUS/Standard does not provide failure in the GTN model. Therefore, ABAQUS/Explicit is used in this analysis. However, mass scaling method is used to eliminate the dynamic effect of the sample when a sufficiently low target time increment is used. For the sake of verification, the force-displacement curves obtained from both analyses before fracture initiation are compared and it is observed that the results are compared well.

Mesh sensitivity is inevitable in FE simulations especially involving localized deformation and fracture. Thus, different meshes are considered in this simulation. Figure 5 shows the FE configuration of an FG tensile test specimen, with a typical mesh consisting of $100 \times 225$ plane strain quadrilateral elements (CPE4R), which are biased to the middle section of the specimen where necking and fracture are expected to happen. The effect of mesh sensitivity on fracture tensile strain will be discussed later in this section.

Figure 6 represents the normalized force $\left(F^{*}\right)$ as a function of the tensile strain $\varepsilon$ for fully base material and the effect of mesh sensitivity on this curve is also included. Force is normalized by the yield stress of FGMs calculated using the rule of mixture and $\varepsilon=\ln \left(1.0+\Delta \mathrm{l} / \mathrm{I}_{\mathrm{o}}\right)$. Initially, the sheet is in the elastic state with increasing small amount of applying strain and the load increases linearly. With a continuous increase in $\varepsilon$, the load increases slowly, and it reaches the maximum value. A sharp knee on the load is observed with further increase of strain. This knee corresponds to the fact that the $f$ reaches $f_{c}$ at the center of the specimen in the middle section and it is when coalescence happens. Eventually, a rapid drop in the force-displacement curve happens immediately after the sharp knee. The rapid drop in force indicates the
Fig. 5 FE configuration of FG tensile test; a FGM layers in ABAQUS; $\boldsymbol{b}$ a typical mesh for the metal sheet with $100 \times 225$ quadrilateral elements (CPE4R in ABAQUS/Explicit)

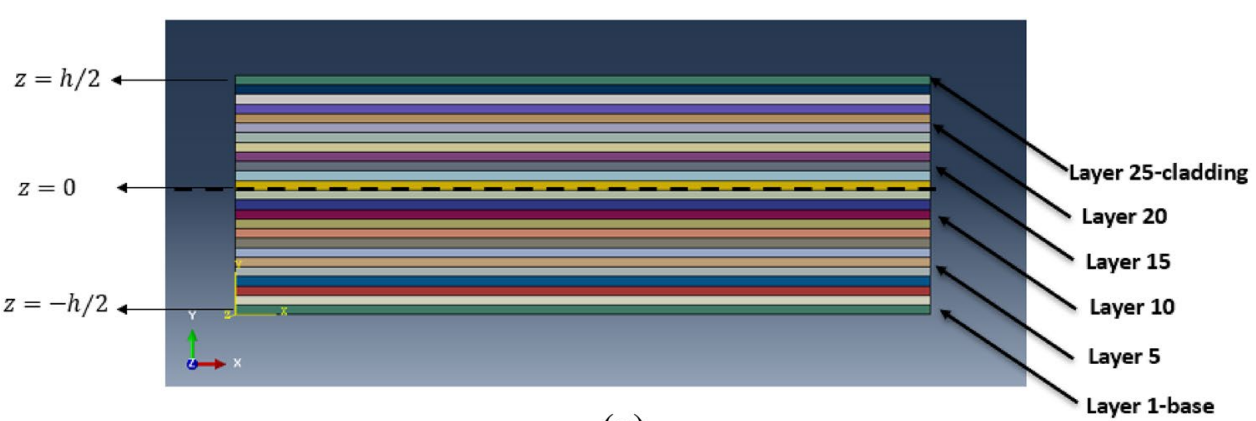

(a)

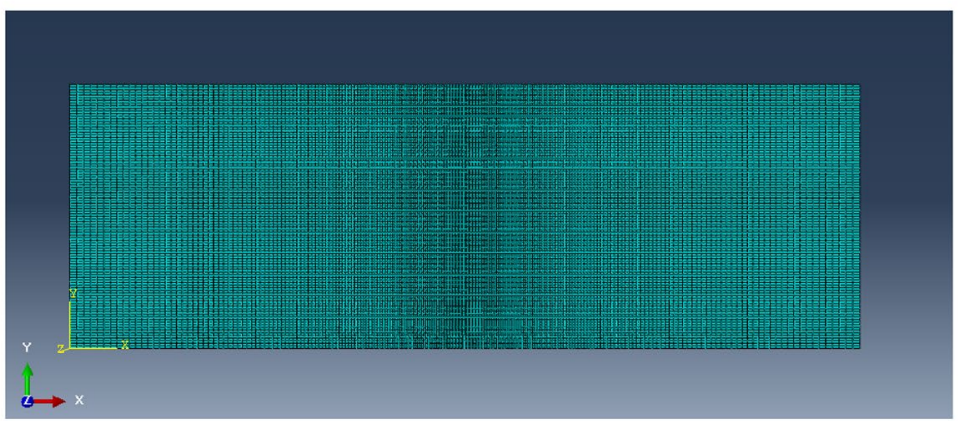

(b) 


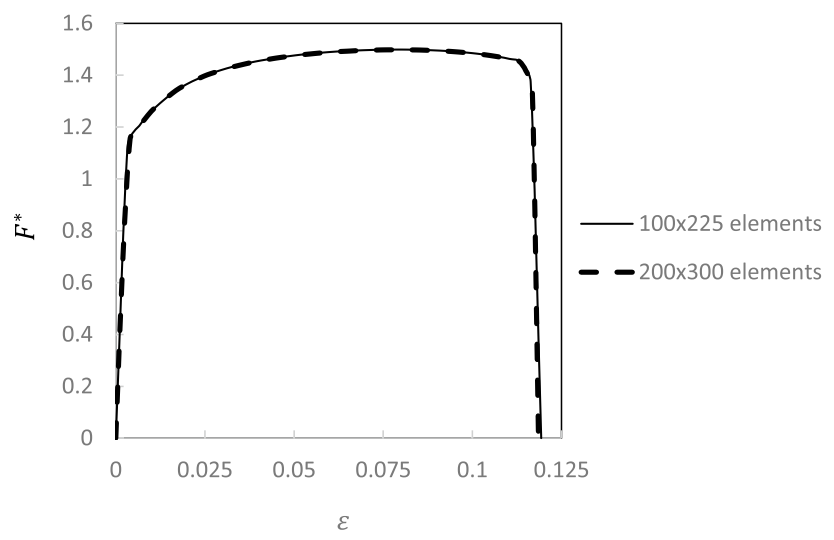

Fig. 6 Effect of mesh sensitivity on normalized force-tensile strain curve

rapid loss of load carrying capacity of the sheet metal because of a burst of void growth and nucleation.

Two different meshes of $100 \times 225$ elements ( 100 elements in the $Y$ direction and 225 elements in the $X$ direction) and $200 \times 300$ elements (200 elements in the $Y$ direction and 300 elements in the $X$ direction) are used for FE configuration of FGMs. The effect of mesh sensitivity on the force-displacement curves is shown in Fig. 6. The figure shows that mesh size has an insignificant effect on the force-displacement curve for the two cases investigated in this study. It is to be noted that the mesh sensitivity on force-displacement curve is reported in other studies frequently such as in Ref. [23] for elastic-plastic problems. However, the fracture strains for FGMs with various grading index $n$ will be calculated in in this sub-section and the mesh sensitivity will be observed.

FGMs with various number of layers for the case $n=1.0$ are simulated, and the effect of number of layers on force-displacement curve are investigated. Results are shown in Fig. 7. It is observed that the force-displacement curves for FGMs with 2 and 5 layers are not converged. Conversely, the results for FGMs containing 10, 25, and 50 layers converge. In this study, the results for FGMs containing 25 layers are presented. Notably, the two-layer FGM is the same as the cladding material with $50 \%$ thickness ratio.

Figure 8 presents the crack patterns in sheet metals for various $n$ values with two different meshes. Two crossed shear bands are developed, and fracture initiates at the center in the middle section of the specimen and propagates along one of the shear bands. The initial geometric symmetry cannot be retained. Instead, the simulations result in a nonsymmetric solution with a single crack as it dissipates low energy. In one specific case ( $n=0.5$ with $200 \times 300$ elements), the crack propagates from the center

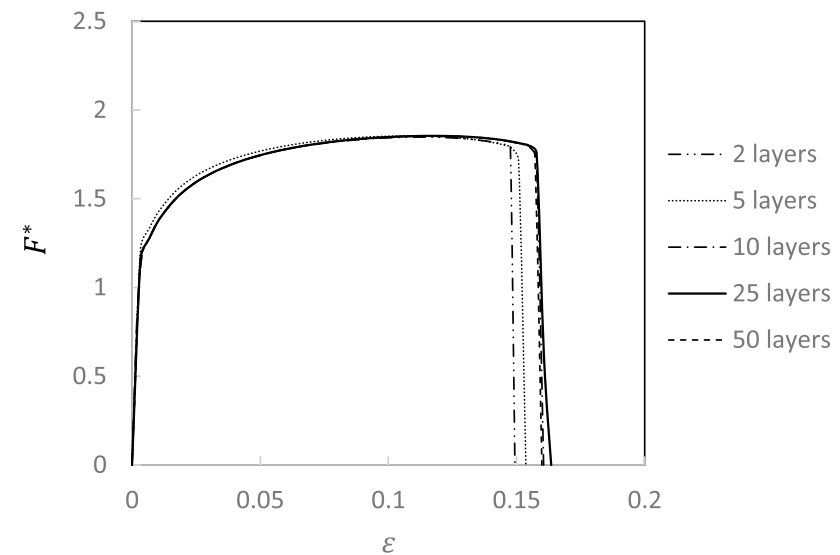

Fig. 7 Effect of number of FGM layers on force-displacement curves

of the specimen and along the two different shears bands, creating a V-shaped crack.

The effect of the $n$ on fracture under plane strain tensile test is studied. Figure 9 shows the effect of $n$ on the normalized force-tensile strain curve and found that the maximum force increases with decreasing $n$ as the effective stress-strain curve increases in FGMs. The elongation in FGMs also increases because the specimens become soft with decreasing $n$. With decreasing $n$, the stress-strain curves in FGM layers are prone to the cladding material, which is softer than the base material.

The necking point in the specimen occurs when the $F^{*}$ reaches the maximum value $\left(d F^{*}=0\right)$ [27]. In Ref. [27], the necking point strains $\left(\varepsilon^{u}\right)$ for cladding materials with various cladding thickness ratios $(\Gamma$ ) are calculated using FEM when $d F^{*}=0$ and compared with those obtained using the Considere rule. It is observed in Ref. [23] that the results calculated using FEM are comparable well to the results obtained using the Considere rule. The results are compared well. The $\varepsilon^{u}$ values for FGMs with various $n$ values are calculated when $d F^{*}=0$ and shown in Table 2 . The results show that $\varepsilon^{u}$ increases with decreasing $n$. The rate of hardening and the stress-strain curve are involved in the calculation of necking point following the Considere rule. The effective stress-strain curves for FGMs are shown in Fig. 3d.

The effect of the $n$ on the normalized minimum crosssectional area $\left(A^{*}=A_{\min } / A_{\mathrm{o}}\right)$ is shown in Fig. 10. $A_{\min }$ is the minimum cross-sectional area, and $A_{o}$ is the initial crosssectional area of the specimen. The $A^{*}$ decreases with decreasing $n$. A decrease in $A^{*}$ indicates a high fracture strain and increased ductility. The tensile fracture strain $\left(\varepsilon^{f}=\ln \left(A_{0} / A_{\text {min }}\right)\right)$ in the middle section of FGM specimens is shown in Table 3. The fracture strain increases with decreasing $n$, and the specimen becomes increasingly ductile. As mentioned previously and in Fig. 4, the $\varepsilon_{N}$ values in the layers of an FGM decrease with increasing $n$. The 


\section{Base material}

Cladding material
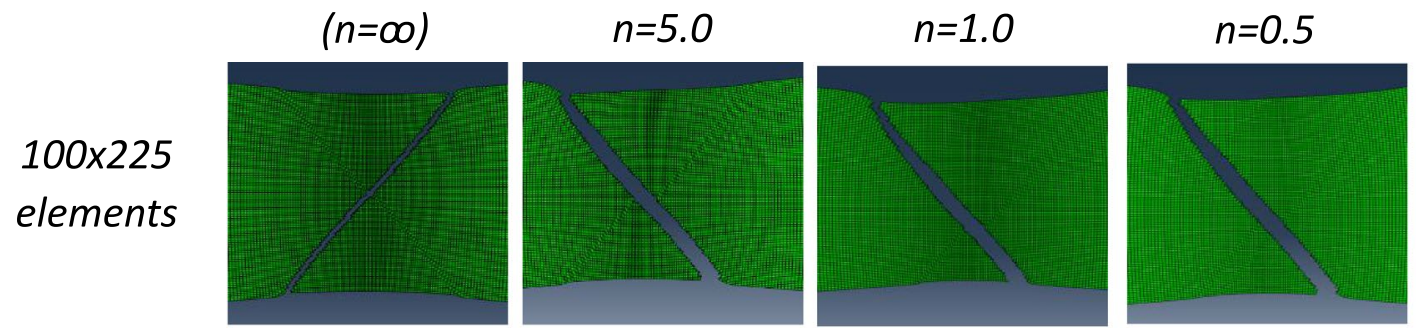

$(n=0)$
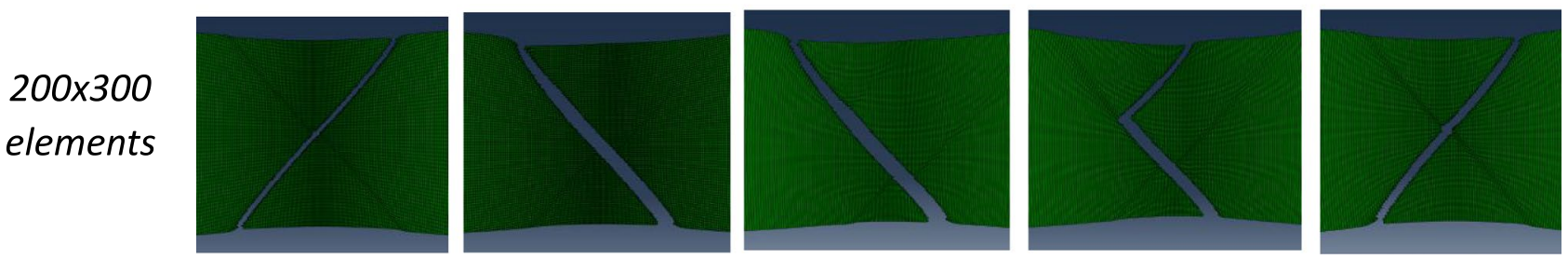

Fig. 8 Predicted fracture modes in FGMs specimen with different values $n$ and number of elements

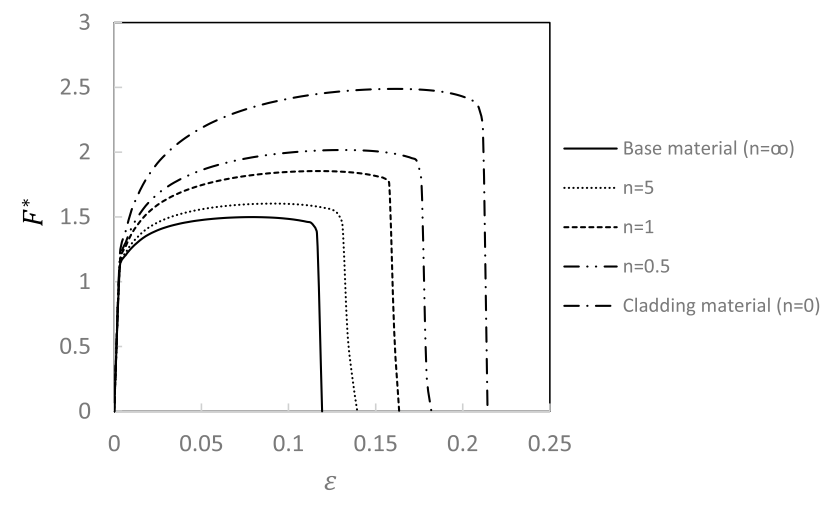

Fig. 9 Normalized force-tensile strain curves of FGMs with various values of $n$

Table 2 Necking points of FGMs with various values $n$

\begin{tabular}{llllll}
\hline$n$ & 0 & 0.5 & 1.0 & 5 & $\infty$ \\
\hline$\varepsilon^{u}$ & 0.162321 & 0.131069 & 0.116233 & 0.092219 & 0.080093 \\
\hline
\end{tabular}

average void nucleating $\left(\varepsilon_{N}\right)$ in the GTN model is a ductile fracture parameter. Fracture is delayed with decreasing $n$. The mesh sensitivity study on the fracture strain is performed, and the fracture strains for two different meshes are compared in Table 3. The mesh has a relatively insignificant effect on fracture strain.

Fracture delay can be explained by presenting how $n$ influences stress triaxiality $\left(\sigma_{H} / \bar{\sigma}\right)$, where $\sigma_{H}=(1 / 3)$ $\left(\sigma_{x x}+\sigma_{y y}+\sigma_{z z}\right)$. Figure 11 presents the $\left(\sigma_{H} / \bar{\sigma}\right)$ at the fracture initiation site for various $n$ values. The figure

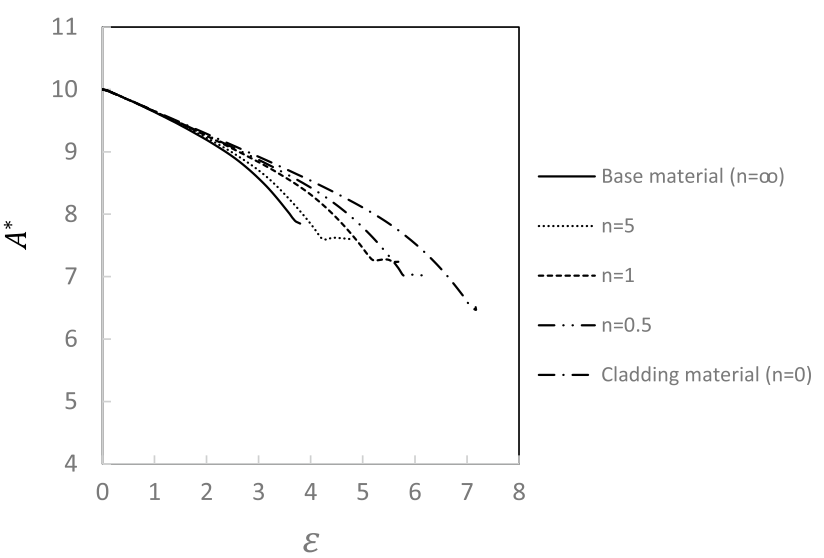

Fig. 10 Normalized minimum cross-sectional area of FGMs with various values $n$

Table 3 Fracture strain $\varepsilon^{f}=\ln \frac{A_{o}}{A_{\min }}$

\begin{tabular}{llllll}
\hline$n$ & 0 & 0.5 & 1.0 & 5 & $\infty$ \\
\hline$\varepsilon^{f}(100 \times 225$ elements $)$ & 0.429 & 0.354 & 0.323 & 0.275 & 0.242 \\
$\varepsilon^{f}(200 \times 300$ elements $)$ & 0.41 & 0.369 & 0.311 & 0.261 & 0.236 \\
\hline
\end{tabular}

shows that the development of $\left(\sigma_{H} / \bar{\sigma}\right)$ slows down with decreasing $n .\left(\sigma_{H} / \bar{\sigma}\right)$ has a significant effect on void growth, and the decrease in $n$ helps delay the fracture. Figure 12 presents the void growth at fracture initiation sites with various $n$ values and demonstrated that void growth slows down with decreasing $n$. Moreover, the average $\varepsilon_{N}$ value of FGM layers decreases with decreased $n$, which helps delay the fracture. 


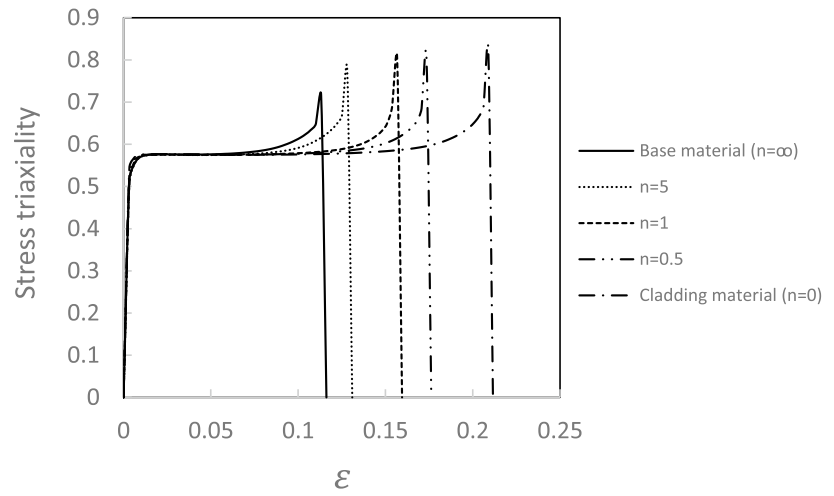

Fig. 11 Stress triaxiality at the fracture site in FGMs with various values $n$

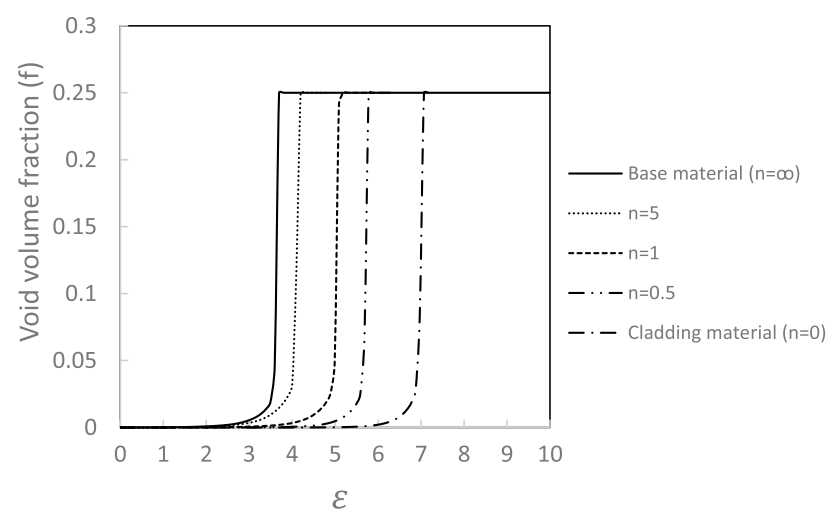

Fig. 12 Total void volume fraction $(f)$ at the fracture site in FGMs with various values $n$

\subsection{Effect of cladding material stress-strain curve}

In this subsection, the effects of cladding material stress-strain curve on necking point and fracture strains are studied. A constant value for grading index $(n=1)$ is considered, and the stress-strain curves and $\varepsilon_{N}$ of every layer in FGMs changes gradually following the power-law distribution presented in Eq. (9). Ductile fracture parameters for every case are the same, and the average $\varepsilon_{N}$ value of FGM layers for every case is 0.4 . The other material properties are the same as studied in a previous subsection. FGMs with various values of $K$ and $\tilde{n}$ for the cladding material stress-strain curves are simulated. These stress-strain curves are shown in Fig. 13. The rate of hardening in the cladding material stress-strain curve changes with changing $\tilde{n}$ and decreases with decreased $\tilde{n}$. Conversely, the rate of hardening in the cladding material stress-strain curve is constant with varying $K$. The necking point and fracture strains of every case are calculated, and results are shown in Figs. 14 and 15, respectively. As shown in Fig. 14, the necking point strain is almost unchanged with changing

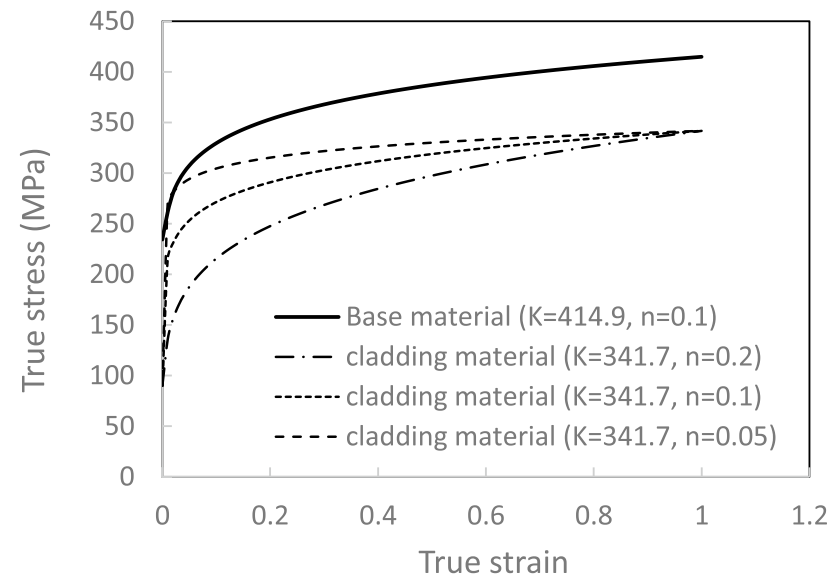

(a) " $\tilde{n}$ " sensitivity

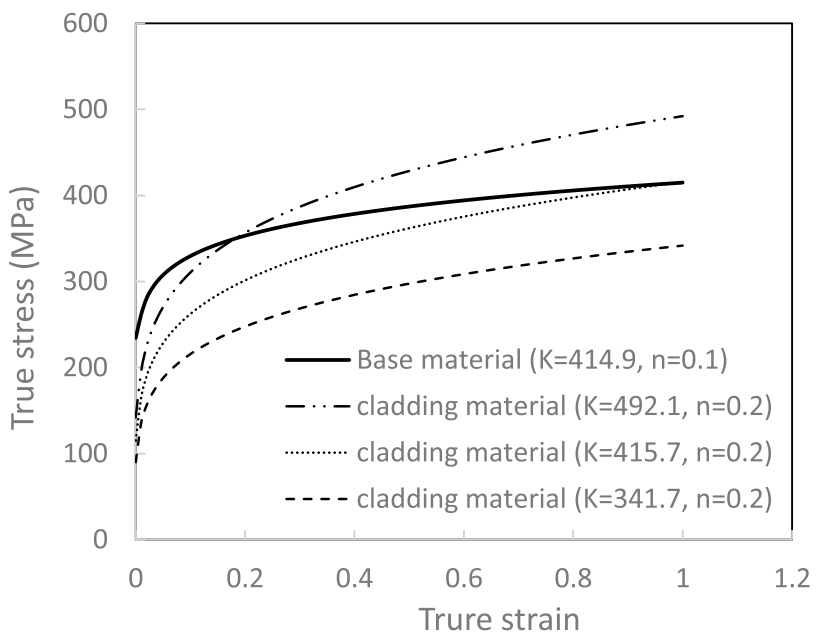

(b) " $K$ " sensitivity

Fig. 13 Stress-strain curves for the matrix and cladding materials with various values of $\mathbf{a}$ " $n$ " and $\mathbf{b}$ " $K$ "

$K$ and increases with increased $\tilde{n}$. Figure 15 shows that the effect of $\tilde{n}$ is more significant than that of $K$ on fracture strain as necking-point strain increased with increased $\tilde{n}$.

\section{Conclusion}

A finite element analysis of plane strain FG tensile tests for sheet metals is carried out to help the researchers for better design and fabrication of FGMs. The lower and upper surfaces are pure hard $(n=\infty)$ and soft $(n=0)$ materials, respectively, in the simulations. Material properties are distributed evenly if $n=1.0$. The effective properties of FGM are prone to the soft material if $n<1.0$. Meanwhile, the effective properties of the FGM are prone to the hard material if $n>1.0$. Decreasing the gradation $n$ increases the fracture strain significantly as the material becomes soft and developed $\sigma_{H} / \bar{\sigma}$ 
Fig. 14 Necking point strains for various values of a " $K$ " and b " $n^{\prime \prime}$
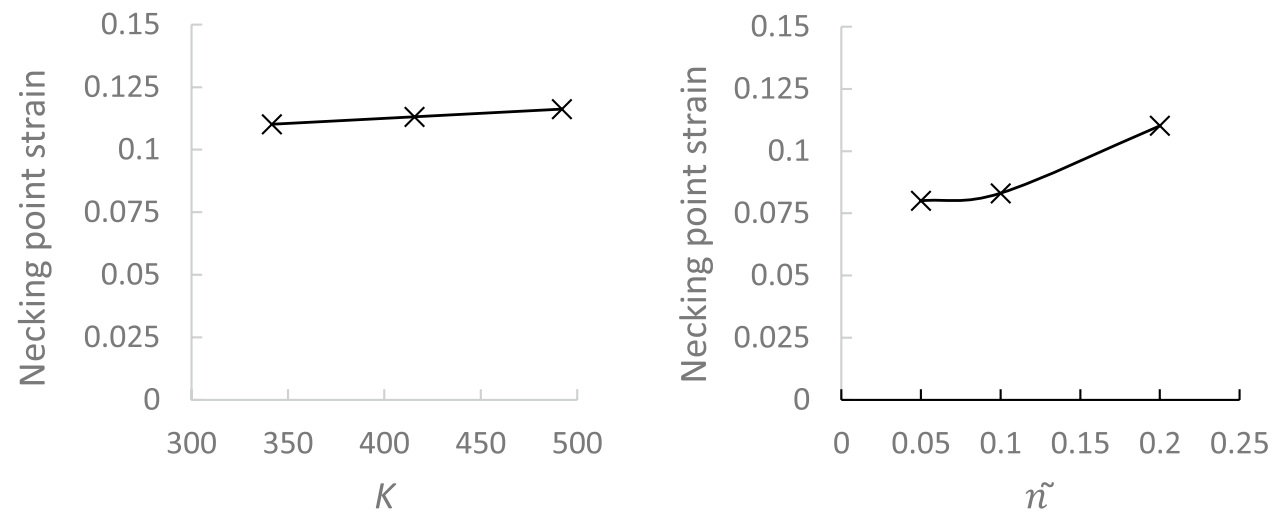

Fig. 15 Fracture strains for various values of $\mathbf{a}$ " $K$ " and $\mathbf{b}$ " $n^{\text {" }}$

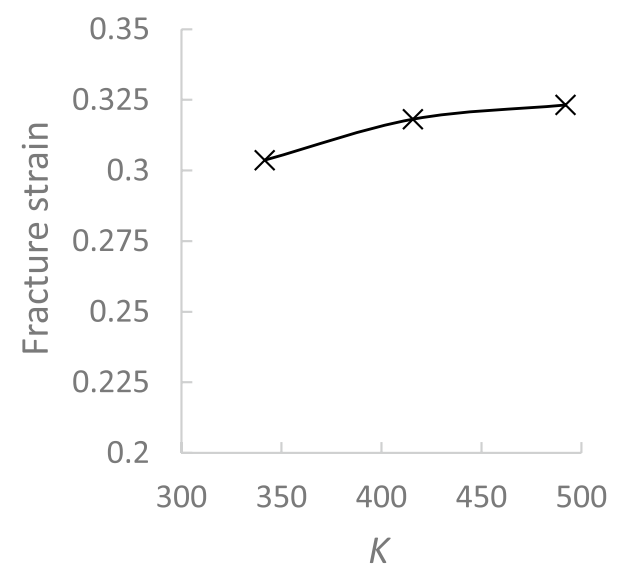

slows down, thereby delaying void growth and microvoid or microcrack coalescence. The necking strain in FGMs is calculated and it is found that it increases when the rate of hardening in the effective stress-strain curve increases for lower values of $n$. The effect of cladding material stress-strain curve on necking point and fracture strain is studied and it is found that the necking point strain and fracture strain are unchanged with changing $K$ and they increase with increasing $\tilde{n}$ in the cladding material stress-strain curve.

Funding The authors declare that they have no known competing financial interests or personal relationships that could have appeared to influence the work reported in this paper.

\section{Complaince with ethical standards}

Conflict of interest The authors declare that they have no conflict of interest.

\section{SN Applied Sciences}

\section{References}

1. Shahzamanian MM, Lloyd DJ, Wu PD, Xu Z (2021) Study of influence of superimposed hydrostatic pressure on bendability of sheet metals. Eur J Mech A 85:104132

2. Partovi A, Shahzamanian MM, Wu PD (2020) Study of influence of superimposed hydrostatic pressure on ductility in ring compression test. J Mater Eng Perform 29:1-10

3. Chen X (2009) Numerical simulation of effects of cladding and superimposed hydrostatic pressure on fracture in metals under tension. Master thesis, McMaster University

4. Lesuer DR, Syn CK, Sherby OD, Wadsworth J, Lewandowski JJ, Hunt WH (1996) Mechanical behaviour of laminated metal composites. Int Mater Rev 41(5):169-197

5. Manoharan M, Ellis L, Lewandowski J (1990) Laminated composites with improved toughness. Technical report. Case Western Reserve University, Cleveland, $\mathrm{OH}$

6. Ritchie R (1988) Mechanisms of fatigue crack propagation in metals, ceramics and composites: role of crack tip shielding. Mater Sci Eng A 103(1):15-28 
7. Kum DW, Oyama T, Wadsworth J, Sherby OD (1983) The impact properties of laminated composites containing ultrahigh carbon (UHC) steels. J Mech Phys 31(2):173-186

8. Kim KJ, Kim D, Choi SH, Chung K, Shin KS, Barlat F, Oh KH, Youn JR (2003) Formability of AA5182/polypropylene/AA5182 sandwich sheets. J Mater Process Technol 139(1-3):1-7

9. Bouaziz O, Brechet Y, Embury JD (2008) Heterogeneous and architectured materials: a possible strategy for design of structural materials. Adv Eng Mater 10(1-2):24-36

10. Suresh S, Mortensen A (1998) Fundamentals of functionally graded materials. The Institute of Materials (IOM) Communications limited

11. Thanh $\mathrm{CL}$, Phung-Van $\mathrm{P}$, Thai $\mathrm{CH}$, Nguyen-Xuan $\mathrm{H}$, Abdel Wahab $M(2018)$ Isogeometric analysis of functionally graded carbon nanotube reinforced composite nanoplates using modified couple stress theory. Compos Struct 184:633-649

12. Barretta R, Faghidian SA, Marotti de Sciarra F, Penna R, Pinnola FP (2020) On torsion of nonlocal Lam strain gradient FG elastic beams. Compos Struct 233:1115502

13. Kordkheili SH, Naghdabadi R (2007) Thermoelastic analysis of a functionally graded rotating disk. J Compos Struct 79(4):508-516

14. Durodola J, Attia O (2000) Deformation and stresses in functionally graded rotating disks. Compos Sci Technol 60(7):987-995

15. Shahzamanian MM, Sahari BB, Bayat M, Mustapha F, Ismarrubie ZN (2010) Finite element analysis of thermoelastic contact problem in functionally graded axisymmetric brake disks. Compos Struct 92(7):1591-1602

16. Shahzamanian MM, Sahari BB, Bayat M, Mustapha F, Ismarrubie ZN (2013) Elastic contact analysis of functionally graded brake disks subjected to thermal and mechanical loads. Int J Comput Methods Eng Sci 14(1):10-23

17. Ke LL, Wang YS (2007) Two-dimensional sliding frictional contact of functionally graded materials. Eur J Mech A 26(1):171-188

18. Gunes R, Aydin M, Kemal Apalak M, Reddy JN (2011) The elastoplastic impact analysis of functionally graded circular plates under low-velocities. Compos Struct 93:860-869
19. Gunes R, Aydin M, Kemal Apalak M, Reddy JN (2014) Experimental and numerical investigations of low velocity impacton functionally graded circular plates. Compos Part B 59:21-32

20. Gologanu M, Leblond JB, Devaux J (1993) Approximate models for ductile metals containing non-spherical voids-case of axisymmetric prolate ellipsoidal cavities. J Mech Phys 41(11):1723-1754

21. Gurson AL (1977) Continuum theory of ductile rupture by void nucleation and growth: Part I-Yield criteria and flow rules for porous ductile media. J Eng Mater Technol 99(1):2-15

22. Tvergaard V, Needleman A (1984) Analysis of the cup-cone fracture in a round tensile bar. Acta Metall 32(1):157-169

23. Chen XX, Wu PD, Embury JD, Huang Y (2010) Enhanced ductility in round tensile bars produced by cladding a ductile ring. Model Simul Mater Sci Eng 18(2):025005

24. Shahzamanian MM, Lloyd DJ, Wu PD (2020) Enhanced bendability in sheet metals produced by cladding a ductile layer. Mater Today Commun 23:100952

25. Chu C, Needleman A (1980) Void nucleation effects in biaxially stretched sheets. J Eng Mater Technol 102(3):249-256

26. Wu PD, Chen XX, Lloyd DJ, Embury JD (2010) Effects of superimposed hydrostatic pressure on fracture in sheet metals under tension. Int J Mech Sci 52(2):236-244

27. Chen XX, Wu PD, Lloyd DJ, Embury JD, Huang Y (2010) Enhanced ductility in sheet metals produced by cladding a ductile layer. $J$ Appl Mech 77(4):041015

Publisher's Note Springer Nature remains neutral with regard to jurisdictional claims in published maps and institutional affiliations. 